\title{
Interplay between eclipses and soft cosmic rays
}

\author{
Shreya Roy, ${ }^{a, *}$ Sayak Chatterjee, ${ }^{a}$ Sayan Chakraborty, ${ }^{a}$ Saikat Biswas, ${ }^{a}$ \\ Supriya Das, ${ }^{a}$ Sanjay K. Ghosh, ${ }^{a}$ Sunil K. Gupta, ${ }^{b, c}$ Atul Jain,,${ }^{b, c}$ \\ Indranil Mazumdar, ${ }^{b, c}$ Pranaba K. Nayak $^{b, c}$ and Sibaji Raha ${ }^{a}$ \\ ${ }^{a}$ Centre for Astroparticle Physics and Space Science, Bose Institute, \\ EN-80, Sector V, Salt Lake, Kolkata 700091, India \\ ${ }^{b}$ Tata Institute of Fundamental Research, \\ Homi Bhabha Road, Mumbai 400005, India \\ ${ }^{c}$ Cosmic Ray Laboratory, \\ Raj Bhavan, Ooty 643001, India \\ E-mail: shreyaroy2509@gmail.com, sayakchatterjee896@gmail.com, \\ sayanc776@gmail.com, saikat@jcbose.ac.in, supriya@jcbose.ac.in, \\ sanjay_k_ghosh@yahoo.co.in, gupta.crl@gmail.com, atul@crl.tifr.res.in, \\ indramazumdar@gmail.com, pranaba@tifr.res.in, sibajiraha@gmail.com
}

\begin{abstract}
Astronomical events such as Solar and Lunar eclipses provide the opportunity for studying the disturbance produced in the atmosphere by these events and their effect on cosmic ray intensity. There are earlier reports on decrease in secondary cosmic gamma ray (SCGR) flux during solar eclipse and enhancement of the same during lunar eclipse. In this paper, we report the results from the measurement of SCGR flux during the solar eclipse on December 26, 2019 and two lunar eclipses on January 31, 2018 and July 27, 2018.
\end{abstract}

$37^{\text {th }}$ International Cosmic Ray Conference (ICRC 2021)

July 12th - 23rd, 2021

Online - Berlin, Germany

\footnotetext{
${ }^{*}$ Presenter
} 


\section{Introduction}

Solar and lunar eclipses are very important astronomical events that provide the opportunity for studying the disturbances produced in the atmosphere due to them and their effect on cosmic ray intensity. The variation of secondary cosmic ray (SCR) flux during solar and lunar eclipses have been studied and reported earlier by several groups of researchers. Most of them observed a decrease in secondary cosmic gamma ray (SCGR) flux during the solar eclipse [1-6] and enhancement of the same during lunar eclipse [7, 8]. Rapid reduction in solar irradiation during a solar eclipse causes many secondary effects on the Earth's atmosphere [9]. The explanation for the decrease in SCGR during solar eclipse given by some groups is that a quasi-periodic pressure wave is set up in the ionosphere by the shadow band in the ozone layer which may, considerably, affect the production of SCGR [10]. Another explanation is that $\pi-\mu$ component production layer of the atmosphere is lowered due to atmospheric cooling during the eclipse which shortens the path (or the time available) for decay of $\pi^{0}$ meson to $\gamma$-rays and $\mu$ meson to $\mathrm{e}^{ \pm}$and induces the changes in relative cosmic ray counts [4]. The drop of SCGR intensity during solar eclipse cannot be explained by atmospheric cooling alone because geophysical disturbances are present at all levels of the atmosphere. The interaction of the cosmic rays in the atmosphere is affected by the weather parameters and solar activities. SCGR is also affected by interplanetary parameters and tidal effects $[8,11]$. However, there is no verified explanation for the increase of SCGR during lunar eclipse. During lunar eclipse, there is a particular geometrical alignment of the Sun, the Earth, and the Moon, which makes the tidal forces very effective, which may be the reason for the observed phenomena. We have measured the SCGR flux in India during two solar eclipses, one on 21 August 2017 passing over North America, and the other on 26 December 2019 passing over South India, and two lunar eclipses, on 31 January and 27 July in 2018 that took place in India. The observation and results of the solar eclipse on 21 August 2017 can be found in the Ref. [12]. The results of the solar eclipse on 26 December 2019 and two lunar eclipses are briefly discussed in the following sections.

\section{Annular solar eclipse of December 2019}

The study of cosmic rays during annular solar eclipse has been done before [6]. Although such measurements has never been performed at high altitudes. With $93.3 \%$ coverage of the Sun, Ooty $\left(11.4^{\circ} \mathrm{N}, 76.6^{\circ} \mathrm{E}\right)$ a hill station in India, became a favourable place for the desired study of cosmic gamma ray variation during the eclipse of December 26, 2019. The eclipse partial began at 8:05:52 am and ended at 11:09:45 am IST. Continuous measurement of SCGR was carried out from 23 December to 29 December 2019 using $\mathrm{NaI}(\mathrm{Tl})$ scintillator detector at the Cosmic Ray Laboratory (CRL, TIFR), Ooty.

\subsection{Experimental setup}

$\mathrm{The} \mathrm{NaI}(\mathrm{Tl})$ detector used in this experiment has a crystal of size 4 in $\times 4$ in $\times 16$ in. The crystal was hermetically sealed inside a thin aluminium casing with a $1 \mathrm{~mm}$ thick white reflecting material placed between the crystal and the casing. The scintillation crystal was optically coupled to photomultiplier tube (PMT), inside the hermetically sealed case. A schematic of the signal 


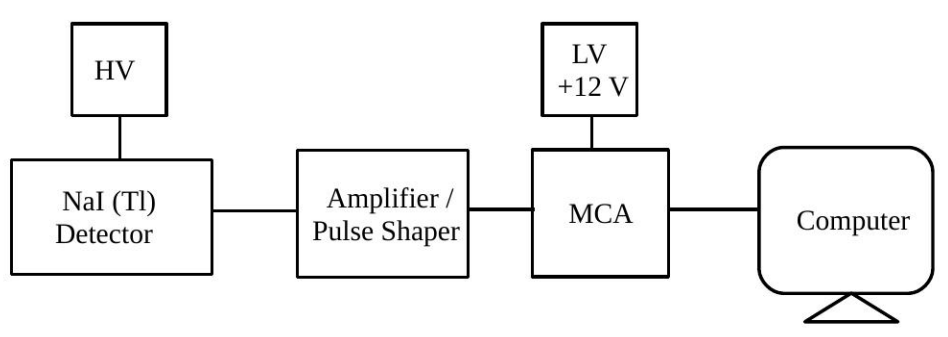

Figure 1: Block diagram of the experimental arrangement

processing electronics is shown in Fig. 1. The signal from the dynodes was fed to a fixed gain charge sensitive pre-amplifier, integrated with the base of the PMT. The pre-amplifier signal was further shaped and amplified using a Spectroscopy Amplifier. The amplifier output was digitized using multi-channel analyzer (MCA). Finally, the data were stored in a personal computer (PC). For each case the energy spectra are stored in PC. The detector was kept outside under the sky without any lead shielding. Temperature, pressure and humidity were continuously monitored at 1 min interval. The detector calibration is done by terrestrial radioactive sources for e.g. ${ }^{40} \mathrm{~K}$ and ${ }^{208} \mathrm{Tl}$ whose energies were known. During the study, the threshold of the detector was set to $125 \mathrm{keV}$. The $\gamma$-ray spectra were stored for 4 minutes and the number of detector signals per seconds was calculated by summing up the counts in all the ADC channels, thereby integrating the entire spectrum and then dividing by the time taken for accumulation of each of the spectrum. In a typical background gamma ray spectrum of the detector, it was found that the detector measures gamma rays in the energy range $125 \mathrm{keV}$ to $15 \mathrm{MeV}$, with about $90 \%$ of the counts within $125 \mathrm{keV}$ to $4 \mathrm{MeV}$. Eliminating the detector noise that generally comes in the first few ADC channels, the total counts in the detector is considered only above $150 \mathrm{keV}$ (i.e. the energy range $150 \mathrm{keV}$ to $4 \mathrm{MeV}$ ).

\subsection{Results and discussions}
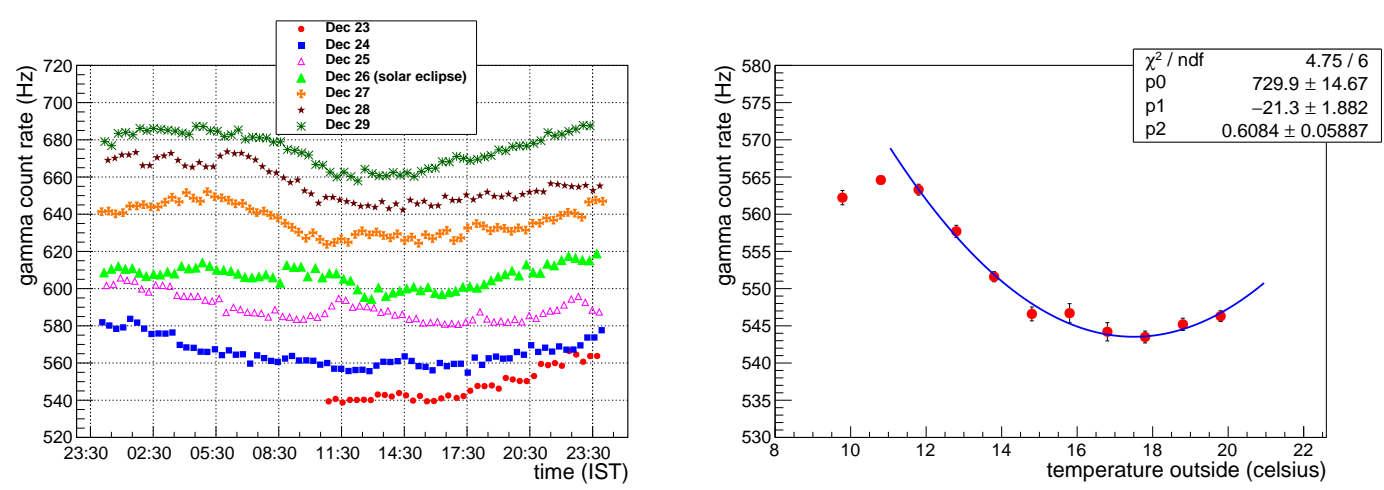

Figure 2: Left:(a) Total counts of secondary cosmic gamma ray per second above $150 \mathrm{keV}$ energy (Error bars are smaller than the marker. Each day's curve is shifted by $20 \mathrm{~Hz}$ w.r.t the previous day's curve for visibility). Right:(b) Correlation between gamma flux and temperature

Fig. 2(a) shows the total $\gamma$-ray counts per second over the detector area measured during $23^{\text {rd }}$ 
to $29^{\text {th }}$ December, 2019. The data points of each day is shifted by $20 \mathrm{~Hz}$ along Y-axis with respect to the previous day's baseline, for clear view of all the points for all days separately in the same canvas. It was found that gamma flux has an inverse correlation with outside temperature, which could be attributed to the temperature dependent gain of $\mathrm{NaI}(\mathrm{Tl})$ detector crystal. Variation of the detector's gain with temperature has been studied earlier and can be found in Ref. [13]. Fig. 2(b) shows the correlation and the curve is fitted by a polynomial function of order 2 . The fit parameters are used to normalise the gamma flux, so that the temperature effect of the crystal is eliminated. The normalised gamma ray count is calculated as,

$$
\text { Normalised gamma ray count rate }=A \times \frac{\text { measured gamma count rate }}{\left(p 0+p 1 . T+p 2 . T^{2}\right)}
$$

where $\mathrm{A}$ is a normalisation constant, p0, p1, p2 are the fit parameters taken from Fig. 2(b) and T is the temperature. Fig. 3(a) shows the temperature normalised gamma count rate for all the days. From Fig. 3(b) a slight decrement of 2.6\% (dip) at 09:44 am 26 December can be observed in the energy range $150 \mathrm{keV}$ to $500 \mathrm{keV}$ during the eclipse. Figure also shows the variation of outside temperature that is uncorrelated with the sudden drop in SCGR. Similar decrements are observed in other energy ranges also and the values of \% decrement are mentioned in Table 1.
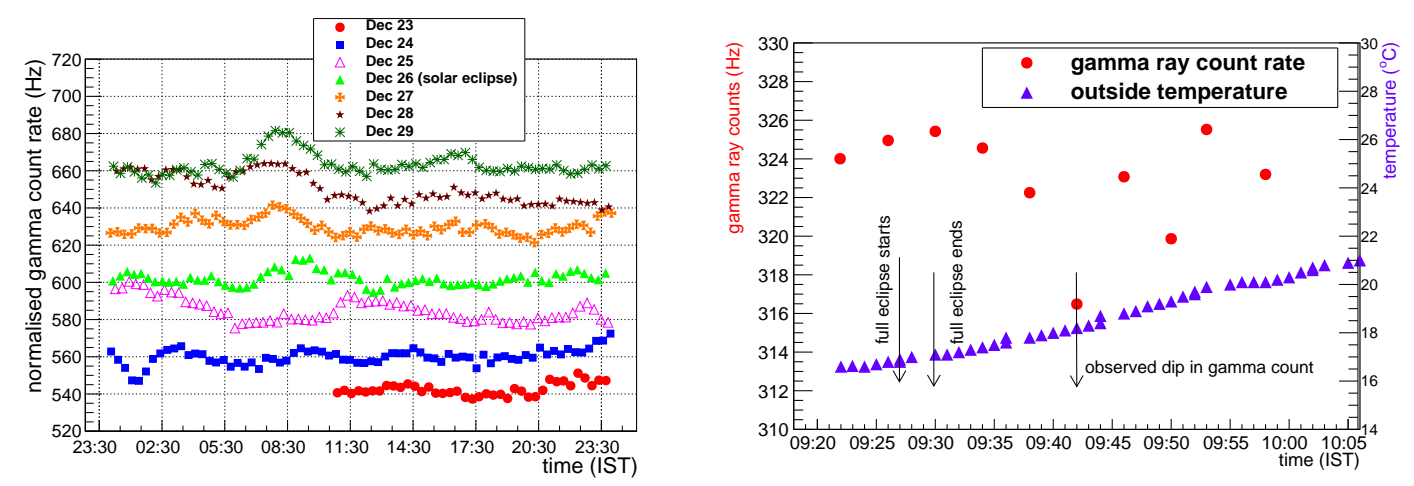

Figure 3: Left:(a) SCGR count rate above $150 \mathrm{keV}$ energy normalised by the temperature effect (Error bars are smaller than the marker. Each day's curve is shifted by $20 \mathrm{~Hz}$ w.r.t the previous day's curve for visibility). Right:(b) Total counts of secondary cosmic gamma ray per second in the energy range $150 \mathrm{keV}$ to $500 \mathrm{keV}$ during the eclipse annularity (Error bars are smaller than the marker).

Generally $\gamma$-ray counts measured in our detector everyday has a decreasing trend during the hours 8 am to 11 am (eclipse hours) Fig. 3(a). To check whether the observed sudden dip in $\gamma$-ray counts are frequent on other days or not, we have done a statistical analysis. The difference in two $\gamma$-ray counts that are time consecutive is taken for all other days and the eclipse day. The difference is filled in a 1D histogram for all the pairs of time consecutive counts. Fig. 4(a) shows the histogram of difference for other days, while Fig. 4(b) shows for the day of eclipse for the energy range 150 to $500 \mathrm{keV}$. Here the means, which if negative signified that the $\gamma$-ray count during 8 am to 11 am has a decreasing trend. For other days this value is -0.02 , whereas during the eclipse this value is -0.12 , more prominently negative. Table shows the value of mean for other energy ranges. From the table it is clear that during the eclipse, the $\gamma$-ray count showed sudden drops, mostly below $1.5 \mathrm{MeV}$, 

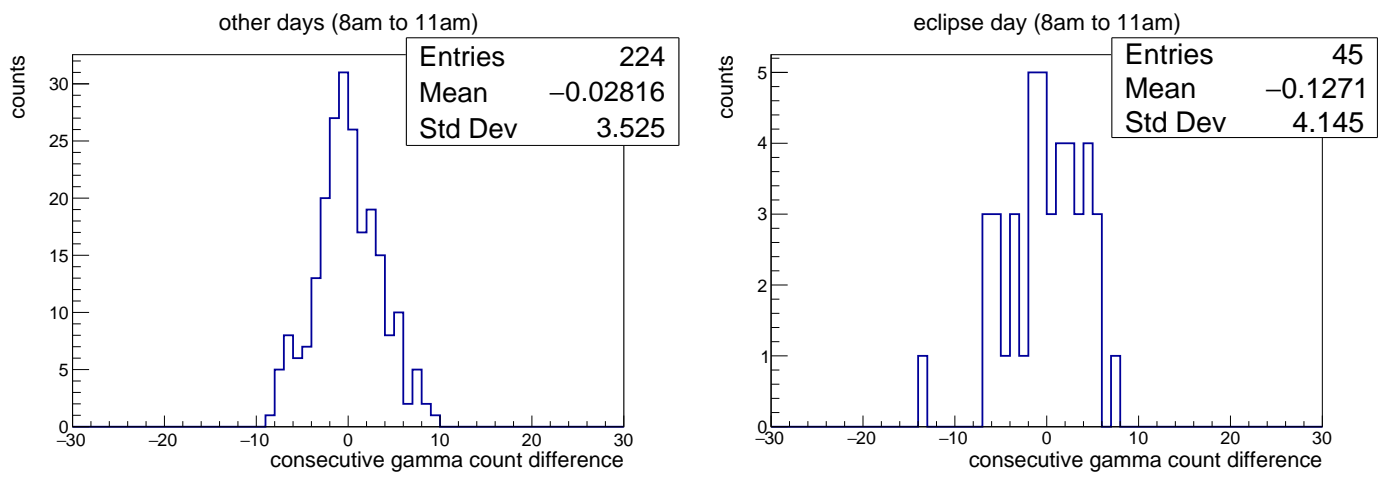

Figure 4: Difference between a pair of time consecutive gamma ray count for other days and the day of eclipse

Table 1: Mean of the differences between a pair of time consecutive gamma count rate for all the data between time 8 am to 11 am

$\begin{array}{llll}\text { Energy range (keV) } & \begin{array}{l}\text { Mean difference } \\ \text { (normal days) }\end{array} & \begin{array}{l}\text { Mean difference } \\ \text { (solar eclipse) }\end{array} & \text { Decrement (\%) } \\ 150-500 & -0.028 & -0.127 & 2.6 \\ 500-1000 & 0.012 & 0.033 & 3.3 \\ 1000-1500 & -0.0003 & -0.007 & 3.8 \\ \text { above } 1500 & -0.001 & -0.01 & 1.2\end{array}$

which is not observed any other day of the week. This result is in agreement with observations by Ref. [6], where they observed a decrement in energy range 1 to $1.5 \mathrm{MeV}$.

\section{Lunar eclipse on January and July 2018}

Enhancement of SCR flux during lunar eclipse was first reported by Ananda Rao in 1967 using Geiger counter [7, 8]. This work became unheeded because during a lunar eclipse, no variation occurs in local weather parameters and hence no variation in SCR is expected. Our aim was to verify their observation and in that direction, we planned to carry out similar experiments during lunar eclipses in India. At the detector laboratory of Bose Institute, Kolkata $\left(22.5^{\circ} \mathrm{N}, 88.3^{\circ} \mathrm{E}\right)$, we measured SCGR flux with $\mathrm{NaI}(\mathrm{Tl})$ detector during the lunar eclipse that took place in the month of January and July of the year 2018. The experimental setup and observations are discussed in the next section.

\subsection{Experimental setup}

The $\mathrm{NaI}(\mathrm{Tl})$ detector used in the present experiment, has a crystal of size $5.1 \mathrm{~cm} \times 5.1 \mathrm{~cm}$. The experimental setup is similar to the one shown in Fig. 1. Measurements were carried out inside a laboratory located at the top floor of the building. The detector was calibrated using standard gamma ray sources ${ }^{137} \mathrm{Cs}(662 \mathrm{keV}),{ }^{60} \mathrm{Co}(1173$ and $1332 \mathrm{keV})$ and ${ }^{22} \mathrm{Na}(511 \mathrm{keV})$ of known energies. Ambient temperature and humidity were kept constant at an average value around $22{ }^{\circ} \mathrm{C}$ and $55 \%$ respectively during the entire duration of measurements using air conditioning system, to minimize 
the variation of the detector gain due to fluctuations in temperature. Continuous measurements were carried out (for three days) starting one day prior to the lunar eclipses till the next day of the eclipses. All the measurements were made keeping the detector on top of a $1 \mathrm{~cm}$ thick lead box such that it was exposed to background radiations from all three directions and the radiation from the bottom (terrestrial radiation) was minimized. No radioactive sources were present nearby.
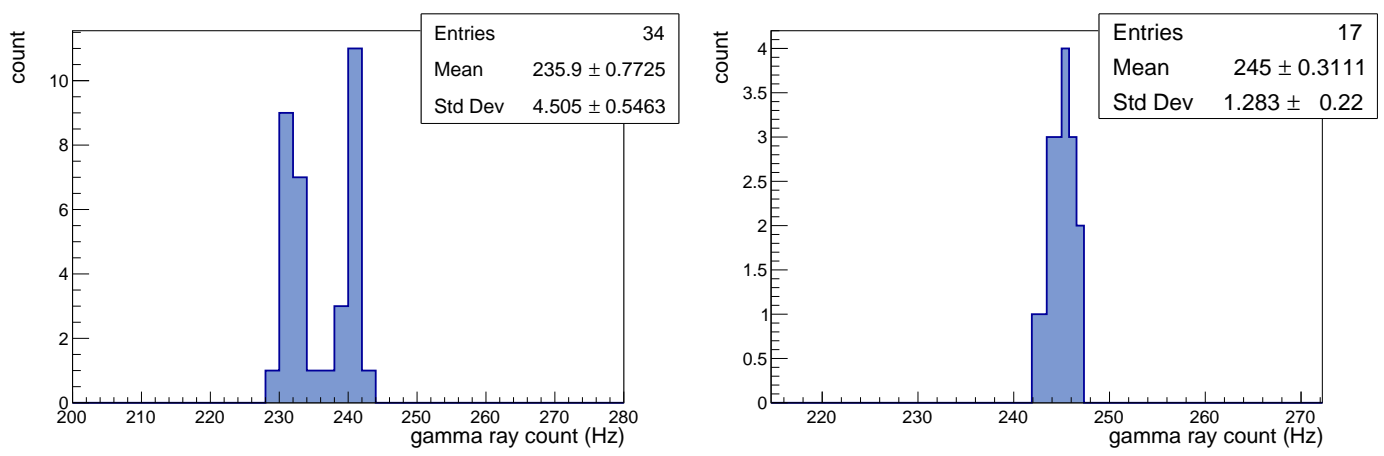

Figure 5: Distribution of gamma count rate from $\mathrm{NaI}(\mathrm{Tl})$ detector during normal days (left panel) and during the lunar eclipse of 31 January 2018 (right panel)
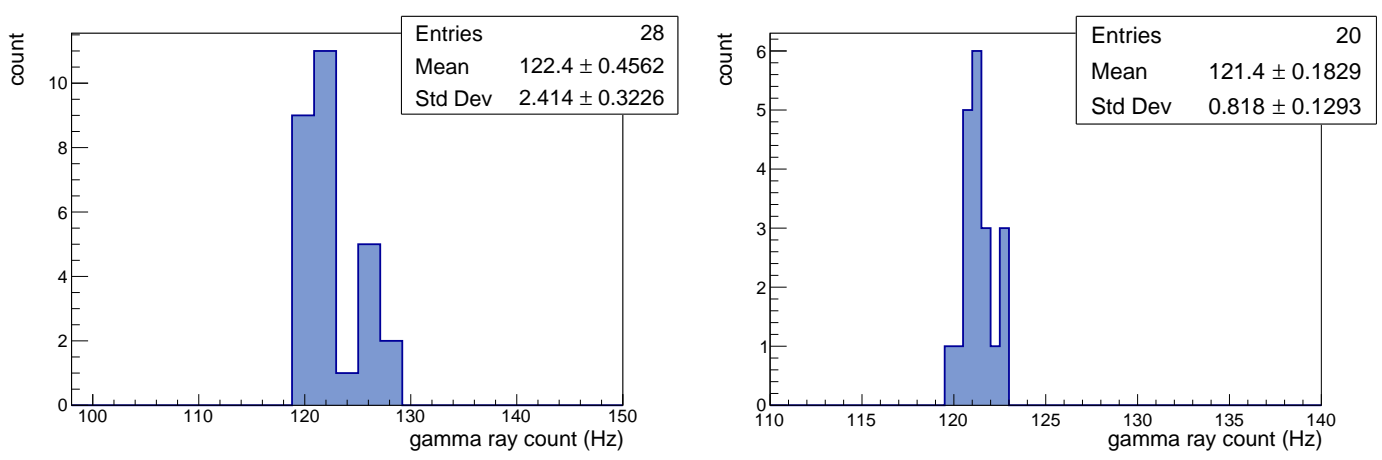

Figure 6: Distribution of gamma count rate from $\mathrm{NaI}(\mathrm{Tl})$ detector during normal days (left panel) and during the lunar eclipse of 27 July 2018 (right panel)

\subsection{Results and discussions}

The lunar eclipse on 31 January 2018, spanned over 4 hours in India starting from 17:18 hr to $21: 38 \mathrm{hr}$ IST. Measurements of SCGR was started one day prior to the day of the lunar eclipse and ended on the next day of the lunar eclipse. $\mathrm{NaI}(\mathrm{Tl})$ detector is used to measure secondary cosmic gamma ray flux in the energy range $25 \mathrm{keV}$ to $3 \mathrm{MeV}$. The distribution of gamma count rate in this energy range during normal days and during the eclipse is shown in Fig. 5. Data points in both the plots are recorded during 17:18 hr to $21: 38 \mathrm{hr}$. The average (mean) gamma count rate during normal days $(17: 18 \mathrm{hr}$ to $21: 38 \mathrm{hr})$ is $(235.9 \pm 0.77) \mathrm{Hz}$, whereas during the lunar eclipse it is $(245 \pm 0.31) \mathrm{Hz}$. This means that there is a slight increment of $3.8 \%$ in the gamma count rate 
during the lunar eclipse. The same experimental setup is used for the measurements during the lunar eclipse of 27 July 2018. It is the longest lunar eclipse of $21^{s t}$ century with a duration of 5 hours (102 minutes of totality) starting from 27 July 22:44 hr to 28 July 03:49 hr. We measured secondary cosmic gamma ray flux in the energy range $50 \mathrm{keV}$ to $3 \mathrm{MeV}$ continuously for 3 days including the eclipse. The distribution of gamma count rate in this energy range is shown in Fig. 6 for normal days and eclipse day. Data during the time interval of the lunar eclipse hours for all three days are only considered. No significant change in gamma flux is observed during the eclipse. Local weather parameters like temperature, pressure and humidity were normal during the lunar eclipse [14]. Space weather also was clear according to the SWPC [15]. The variation of detector gain is only about $0.6 \%$ throughout the measurement. Although we observed a mild increase ( $3.8 \%$ ) in the SCGR over the baseline measurement during the lunar eclipse in January, no such variation was observed during the eclipse of July 2018. Measurements must be repeated in future to reach a consensus on the variation of SCGR during lunar eclipses.

\section{Summary}

We have systematically measured the SCGR flux using a NaI(Tl) scintillator detector during the annular solar eclipse on 26 December 2019. Analysis were done for different energy regions to find out in which energy range the SCGR decrement is dominant. During the annular solar eclipse, we have observed a SCGR flux decrement of $2.6 \%$ in the energy range 150-500 keV and $3.3 \%$ in the energy range $0.5-1 \mathrm{MeV}$ and $3.8 \%$ in the energy range 1-1.5 MeV. Such a kind of experiment has been carried out at high altitude ( $2240 \mathrm{~m}$ above sea level) for the first time. SCGR flux is influenced by various factors such as geomagnetic field, interplanetary parameters, ionospheric parameters and space weather. Checking the correlation of these factors with the observed variation in SCGR during the eclipse is in future plan. A detailed investigation must be carried out in the future for a deep understanding of the phenomenon and its consequences. We have also reported SCGR flux variations during two remarkable lunar eclipses of the same year (2018) in India. Weather parameters are also observed. An enhancement of 3.8\% is observed in the SCGR flux of energies above $25 \mathrm{keV}$ during the lunar eclipse of January. No changes in SCGR is observed during the lunar eclipse of July which is not consistent with earlier results [8]. Although these observations need explanation which cannot be given based only on the present measurements. Such experiments must be repeated in future eclipses so as to substantially improve on the statistics.

\section{Acknowledgement}

S. Roy acknowledges support from the Department of Atomic Energy, Government of India through a Raja Ramanna Fellowship. This work is partially supported by the research grant SR/MF/PS-01/2014-BI from DST, Govt. of India, the research grant of CBM-MuCh project from BI-IFCC, DST, Govt. of India and IRHPA (Intensification of Research in High Priority Areas/Sanction No. IR/S2/PF.01/2011) scheme.

\section{References}

[1] Nayak, P. K., et al., (2016) Astropart. Phys. Volume 72, 55-60 
[2] Bhattacharya, R., et al., A (2010a):Current Science, 98, 1609-1614

[3] Chintalapudi, S. N., et al., (1997), http://hdl.handle.net/2248/6022

[4] Bhattacharyya, A., et al., (1997)Astrophys. Space Sci. 250: 313.

[5] Nayak, P. K., et al., (2010) Astropart. Phys. Vol. 32, Issue 6

[6] Bhaskar, A., et al., (2011) Astropart. Phys. Vol. 35, Issue 5

[7] Ananda Rao, J. N., (1967), Phys. Lett. A, 25, 74-74

[8] Raghav, A., et al., (2013), Journal of Geophysical Research: Space Physics, Vol. 118

[9] Eckermann, S. D., et al., (2007) J. Geophys. Res, Vol. 112, D14105

[10] Antonova, V. P., et al., Bull. Russ. Acad. Sci. Phys. (2007) 71: 1054.

[11] Dorman, L. I., (2009), Astrophysics and Space Science Library, Springer

[12] S. Roy et al., Astrophys Space Sci 365, 172 (2020)

[13] Ianakiev, K. D., et al., (2006) DOI: 10.1016/j.nima.2009.02.019

[14] https://www.timeanddate.com/weather/india/kolkata

[15] http://www.spaceref.com/news/viewsr.html?pid=51598 .... 